\title{
Impuestos para el control del tabaquismo: las experiencias de Brasil y México
}

\author{
Belén Sáenz de Miera-Juárez, M en Econ, ${ }^{(I)}$ Roberto Iglesias, Dr en Econ. ${ }^{(2)}$
}

\author{
Sáenz de Miera-Juárez B, Iglesias $\mathbf{R}$. \\ Impuestos para el control del tabaquismo: \\ las experiencias de Brasil y México. \\ Salud Publica Mex 2010;52 supl 2:S172-SI85.
}

\begin{abstract}
Resumen
Este trabajo pretende analizar los avances, obstáculos y retos en materia de impuestos a los cigarrillos en Brasil y México. En años recientes ambos países han incrementado estos impuestos y han incorporado alícuotas fijas, lo cual permite maximizar el efecto del aumento de los impuestos sobre los precios. Sin embargo, parece que estas medidas no se han aprovechado en toda su potencialidad. Tres recomendaciones surgen de estas experiencias: I) las alícuotas fijas deben representar un porcentaje significativo del precio final; 2) deben ajustarse regularmente de acuerdo con la inflación acumulada; y 3) deben ajustarse para compensar el crecimiento del ingreso personal. También es importante que los impuestos a los sustitutos legales sean similares a los de los cigarrillos. En el caso de los sustitutos ilegales, la cuestión es reducir los incentivos y limitar la facilidad con que se realiza su distribución y venta.Adecuaciones en las legislaciones nacionales y cooperación internacional constituyen elementos clave para lograrlo.
\end{abstract}

Palabras clave: tabaco; impuestos; elasticidad; Brasil; México
Sáenz de Miera-Juárez B, Iglesias R.

Taxation and tobacco control:

the cases of Brazil and Mexico.

Salud Publica Mex 2010;52 suppl 2:SI72-SI85.

\begin{abstract}
This study analyzes the achievements, obstacles and challenges related to cigarette taxes in Brazil and Mexico. Both countries have increased cigarette taxes in recent years and have included a specific tax, which allows maximizing the impact of tax increases on prices. However, it seems they have not taken full advantage of these measures. Three recommendations follow from these experiences: I) specific taxes must represent a significant percentage of final prices; 2) specific taxes must be regularly adjusted to keep pace with cumulative inflation; and 3) specific taxes must be adjusted to compensate increases in personal income. It is also important to consider that taxes on legal substitutes of cigarettes must be similar to cigarette taxes. As for illegal substitutes, the issue is to reduce the incentives for illicit trade and to make illegal transactions more difficult. Modifications to national legislations and international cooperation constitute key elements to achieve this.
\end{abstract}

Key words: tobacco; taxes; elasticity; Brazil; Mexico
E uso de tabaco es la principal causa de muerte prevenible. Se estima que poco más de 5 millones de las muertes de adultos de 30 años o más ocurridas en 2004 mundialmente pueden atribuirse a este factor de riesgo, de las cuales alrededor de $5 \%$ corresponden a países de ingresos medios y bajos del continente americano, es decir, a países de América Latina y el Caribe. ${ }^{1}$

Desde su adopción por la Asamblea Mundial de la Salud en mayo de 2003, el Convenio Marco de la OMS para el Control del Tabaco (CMCT) ha sido un

(I) Departamento de Investigación sobre Tabaco, Instituto Nacional de Salud Pública. México, DF, México.

(2) Universidad Católica de Río de Janeiro.Alianza del Control del Tabaquismo. Río de Janeiro. Brasil.

Fecha de recibido: 26 de marzo de 2010 - Fecha de aceptado: 2 de julio de 2010

Solicitud de sobretiros: Mtra. Belén Sáenz de Miera Juárez. Departamento de Investigación sobre Tabaco, Instituto Nacional de Salud Pública. 7a. cerrada de Fray Pedro de Gante 50, col Sección XVI. 14000 Delegación Tlalpan, México, Distrito Federal, México.

Correo electrónico: bsaenzdemiera@correo.insp.mx 
instrumento clave en la lucha contra la epidemia del tabaquismo. ${ }^{2}$ De los 168 países que han suscrito el CMCT, la mayoría lo han ratificado, aunque su grado de avance en el cumplimiento de las diversas disposiciones que en él se contemplan difiere de manera importante.,4

El primer reporte de la OMS sobre la situación de la epidemia del tabaquismo publicado en 2008 establece seis medidas, que si fueran implementadas de manera simultánea, podrían ayudar al cumplimiento del CMCT. ${ }^{3}$ Dichas medidas son: 1) vigilar el consumo de tabaco, 2) proteger a la población del humo de tabaco, 3) ofrecer ayuda para el abandono del tabaco, 4) advertir sobre los peligros del tabaco, 5) cumplir las prohibiciones de la publicidad, promoción y patrocinio, y 6) aumentar los impuestos al tabaco. De todas ellas, el incremento de los impuestos de forma periódica de acuerdo con la inflación y por encima del crecimiento del poder adquisitivo de los consumidores se considera la intervención más efectiva para reducir el consumo de tabaco. ${ }^{3}$

El incremento de los impuestos opera como sigue. Los impuestos forman parte importante del precio de los productos de tabaco, por lo que su incremento se traduce en un aumento de los precios. Los individuos, al enfrentar precios más altos, responden con la reducción de su consumo. ${ }^{5,6}$ Debido a que la reducción en el consumo es menor que el aumento de los precios, la recaudación fiscal proveniente de los productos de tabaco aumenta. Sin embargo, el ingreso disponible también es un determinante del consumo de tabaco: cuando éste se incrementa, los consumidores aumentan su consumo. ${ }^{5,6}$ Por esto es importante que el alza de los impuestos sea por encima del crecimiento del ingreso real. También es importante que los impuestos se ajusten periódicamente de acuerdo con la inflación, para evitar que los productos de tabaco se vuelvan relativamente más baratos.

El objetivo de este texto es revisar los avances, obstáculos y retos en materia de política fiscal aplicable a los cigarrillos en Brasil y México. Ambos países, al igual que la mayoría de los países latinoamericanos, ha ratificado el $\mathrm{CMCT}^{*}$ y en ellos se concentra más de la mitad de la población de América Latina y una gran parte de los fumadores de la región. De acuerdo con encuestas nacionales recientes, 17.5 y $18.9 \%$ de la población adulta de Brasil y México, respectivamente, fuma en la actualidad. 7,8

\footnotetext{
* El sitio en internet de la Organización Panamericana de la Salud tiene información detallada sobre los países de la región que han firmado y / o ratificado el CMCT. Algunos de los países de América Latina que no han ratificado el CMCT son Argentina, Costa Rica, El Salvador y Nicaragua. Brasil y México ratificaron el CMCT en noviembre de 2005 y mayo de 2004, respectivamente.
}

En las primeras dos secciones se analiza la evolución del consumo de cigarrillos en Brasil y México y su relación con los precios e impuestos vigentes, así como los principales obstáculos y desafíos de sus políticas tributarias. En la última sección se discuten las experiencias presentadas y se hacen recomendaciones.

\section{El caso brasileño}

\section{Evolución del consumo por adultos (1991-2009)}

En la figura 1 pueden observarse tres fases en la evolución del consumo anual de cigarrillos por adulto: una fuerte reducción del consumo total entre 1991 y 2000; fluctuación de las cantidades anuales consumidas, pero alrededor de un nivel superior al de 2000, a partir de ese año y hasta 2004; y, por último, reducción sostenida del consumo legal entre 2005 y 2009. El consumo anual de cigarrillos legales por adulto era de 1625 unidades en 1991, llegando a menos de 700 unidades en 2009. La oferta de cigarrillos sin impuestos internos o ilegales se expandió entre 1992 y 1998, llevando el consumo anual por adulto de esos productos de 60 unidades en 1992, hasta 500 unidades en 1998, según las estimaciones disponibles. ${ }^{*}$ Posteriormente y hasta 2005 , este consumo se mantuvo en niveles próximos a 400 unidades por año, observándose consumos menores en 2006 y 2007.

La evolución del consumo por adulto desde 1991 se vincula con dos variables claves: la política de control del tabaquismo que comprende medidas no relacionadas con precios y la evolución del precio real de los cigarrillos. $^{\ddagger}$ En relación con la primera, Brasil avanzó en forma consistente a partir de mediados de la década de los noventa: ${ }^{\S}$ se aprobó la legislación que restringía la propaganda de cigarrillos, se colocaron advertencias más severas en las cajetillas, se ampliaron las restriccio-

\footnotetext{
* En Brasil no hay cálculos oficiales del consumo anual total de cigarrillos, de los cuales pueda deducirse el consumo aparente legal y la cifra de consumo ilegal. Existen estimaciones extraoficiales, originadas principalmente en informaciones de la industria, que pueden considerase sesgadas (pues la industria tiene interés en sobreestimar el volumen del mercado ilegal, para evitar aumentos de impuestos o solicitar su disminución). En el caso brasileño estas informaciones son normalmente cualificadas por el análisis que hacen los expertos oficiales, pero eso no implica la eliminación completa del sesgo introducido por la industria.

* Las estimaciones econométricas de la demanda de cigarrillos indican que el ingreso real no es significativo como variable explicativa del consumo por adulto en el periodo 1991-2005, pero ese resultado puede estar influenciado por circunstancias macroeconómicas de esos años. ${ }^{9}$

\& Las primeras medidas de restricción se implementaron en la segunda mitad de los años ochenta, cuando comenzó el trabajo activo de control del tabaquismo. En la segunda mitad de los años noventa se avanzó con la legislación, las campañas nacionales y la creación de una red nacional de control del tabaquismo.
} 


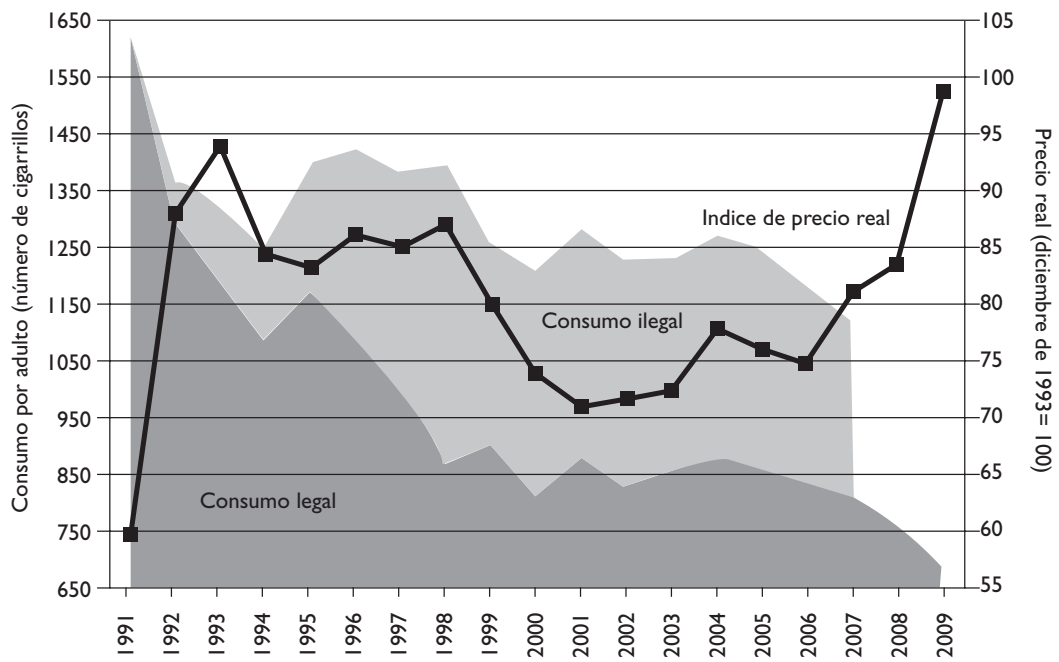

Fuentes: Precio real= precio promedio de la cajetilla con 20 cigarrillos en el Índice Nacional de Precios al Consumidor Amplio (IPCA)/IPCA - Instituto Brasileño de Geografía y Estadística (IBGE). Consumo legal por adulto=(producción + importaciones - exportaciones)/población de más de 15 años - Receita Federal do Brasil (RFB), Ministerio de Industria y Comercio e IBGE. Consumo ilegal por adulto - RFB.

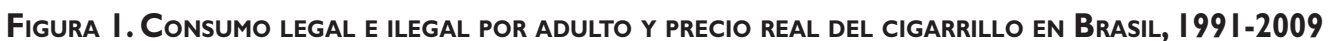

nes para fumar en lugares de uso público, se realizaron campañas públicas coordinadas por el Programa Nacional de Control del Tabaquismo y, en la presente década, se colocaron pictogramas en las cajetillas. Todas estas medidas comenzaron a tener mayores impactos hacia el final de la década de los noventa, y fueron responsables, en la presente década, del bajo crecimiento del consumo por adulto, el cual tuvo lugar a pesar de la caída del precio real entre 1998 y 2005. ${ }^{9}$ En relación con el precio real, su evolución estuvo asociada con desarrollos y eventos del mercado ilegal, tanto cuando fue elevado por decisiones de la industria, entre 1992 y 1998, como cuando la política tributaria contribuyó a su reducción en los primeros años de la década actual, con el objetivo de frenar el crecimiento de la oferta de cigarrillos de contrabando.

\section{Alza del precio del cigarrillo y mercado ilegal}

En los años noventa, después de la eliminación de los controles gubernamentales sobre el precio al consumidor de la cajetilla de cigarrillos, la industria local aumentó el precio de la misma por encima de la inflación, sin que hubiese habido alteraciones en la política tributaria. Este

\footnotetext{
* En la década de los noventa, hasta mayo de 1999, los cigarrillos pagaron los mismos impuestos que los demás bienes industriales, con la diferencia de que la alícuota efectiva del impuesto sobre productos industriales (IPI) era de $42.5 \%$ del precio de venta al consumidor, es decir, mayor a las otras. No hubo cambios en esta tasa durante dicho periodo. La estrategia de la industria de aumento del precio real al consumidor resultó en un crecimiento de la recaudación real del IPI.
}

aumento del precio real redujo la capacidad adquisitiva de los salarios en términos de cigarrillos, forzando la disminución del consumo de cigarrillos legales. La relación entre cantidades demandadas por adulto en el mercado legal y el precio real en Brasil se ha estimado en - 0.48 , lo cual implica que un aumento de $10 \%$ del precio real reduce la cantidad consumida en $4.8 \%$, después de aproximadamente tres trimestres del incremento del precio. ${ }^{9}$

La reducción del poder adquisitivo de los salarios en términos de cigarrillos legales forzó a los más pobres a buscar sustitutos más baratos. La figura 2 presenta la relación entre el salario mínimo y el precio promedio de una cajetilla, es decir, el número de cajetillas que se pueden comprar con un salario mínimo. El efecto del aumento del precio de 1993 puede observarse en el hecho de que los trabajadores podían comprar aproximadamente 150 cajetillas en 1992, mientras que en el año siguiente su poder de compra se redujo a un tercio. En países de ingresos bajos y medios, con una distribución muy desigual del ingreso como en Brasil, la relación entre el ingreso disponible de los estratos más pobres (el salario mínimo en este caso) y el precio promedio es fundamental para determinar el consumo total de cigarrillos y la división del consumo entre productos legales e ilegales. Una caída de esta relación disminuye el consumo total, pero puede aumentar la participación de los cigarrillos más baratos, sin impuestos o contrabandeados, $\mathrm{u}$ otros sustitutos, como tabaco de armar, en el consumo total.

¿Cuál fue la lógica de aumentar significativamente el precio real de los cigarrillos tradicionales por parte de los productores y reducir la capacidad adquisitiva de los 


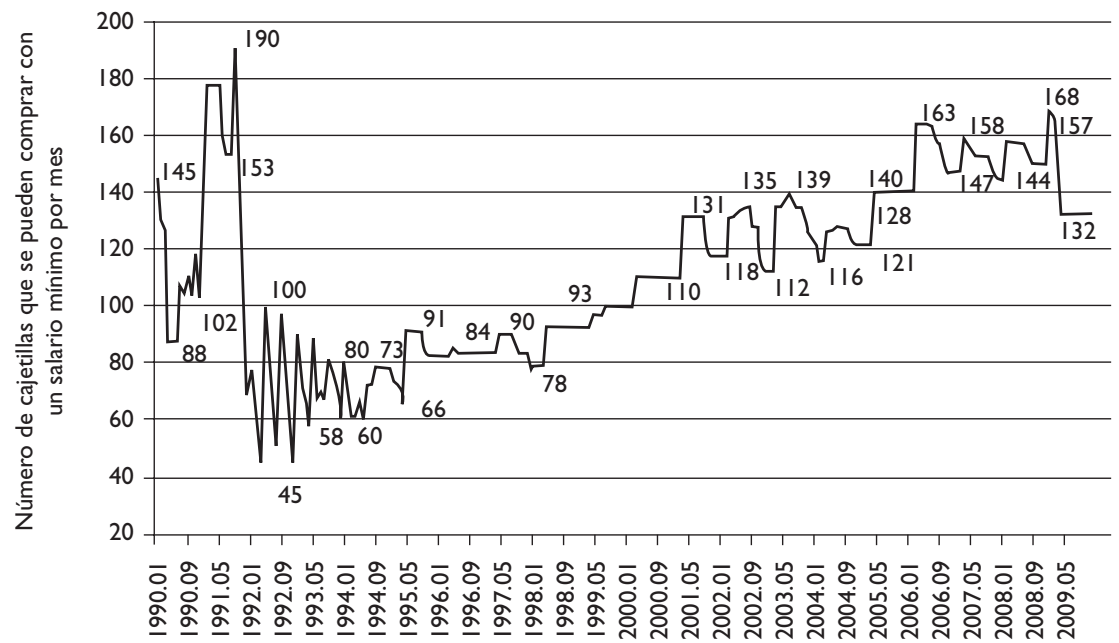

Fuentes: Salario mínimo - Instituto de Pesquisa Económica Aplicada (IPEADATA). Precio promedio de la cajetilla - IBGE

Figura 2. Número de cajetillas de cigarrillos que Se compran con un salario mínimo mensual en BrasiL, 1990 -2005

ingresos de los más pobres sin mediar un aumento de la presión tributaria? Seguramente no fue reducir el consumo total, pues esto atenta contra su masa de beneficios. La respuesta parece haber sido crear demanda para sustitutos más baratos. ¿Por qué crear mercado para cigarrillos baratos? Como se verá, el objetivo final era cambiar la carga tributaria sobre el cigarrillo brasileño.

Para ofrecer cigarrillos más baratos a los más pobres, la industria empezó a exportar enormes cantidades de cigarrillos a Paraguay (varias veces superiores al consumo anual total de ese país). Estos cigarrillos, que no pagaban los impuestos internos de Brasil, eran, por lo tanto, más baratos en el país vecino, y se reintroducían después a Brasil, a través de una red de organizaciones criminales que se fue formando y consolidando en los años noventa. A pesar de los márgenes de intermediación de los contrabandistas, el cigarrillo volvía a los suburbios de las grandes capitales brasileñas más barato que el producto similar con todos los impuestos domésticos.

No existen evidencias de conexiones entre laindustria legal y la red de contrabandistas, pero es evidente que la oferta disponible puesta en Paraguay, a precios muy inferiores a la brasileña legal, y la falta de controles, en aquel momento, de los flujos de cigarrillos a ambos lados de la frontera, creó oportunidades y facilitó la realización del contrabando.* En otras palabras, el mercado ilegal fue creado por una estrategia de las empresas instaladas

\footnotetext{
* Iglesias ${ }^{10}$ explica que las exportaciones legales sólo podían ser hechas por empresas registradas en la Receita Federal, por lo tanto, por sociedades comerciales e industriales debidamente autorizadas y
}

en Brasil, principalmente por British American Tobacco (BAT) y Phillips Morris, con dos instrumentos: aumento del precio real de los cigarrillos domésticos y exportación a Paraguay de cantidades superiores al consumo total anual de ese país. ${ }^{\ddagger}$

En 1998, el gobierno brasileño colocó un impuesto de exportación a los cigarrillos brasileños destinados a Paraguay y Uruguay, lo que eliminó la rentabilidad de la triangulación de las empresas brasileñas y los contrabandistas con base en Paraguay. Pero, lamentablemente, ya era tarde, pues las oportunidades de lucro

fiscalizadas, entre las cuales se encontraban las grandes empresas. ${ }^{10}$ Este dato es hoy sigiloso para el público y los investigadores académicos, pero nunca lo fue para las autoridades del Poder Ejecutivo. En los años noventa, cuando los paquetes eran encontrados en territorio brasileño $\sin$ los impuestos internos, las empresas productoras brasileñas alegaban falsificación de la industria paraguaya, para evitar con ese argumento las multas y obligaciones que la ley brasileña exigía. Pero ese argumento no tenía consistencia, pues, en esa época, la industria del país vecino era muy pequeña y sin capacidad para producir esa cantidad de cigarrillos. La posibilidad de esta defensa de la industria revela que cometían una infracción al exportar: no colocaban en la cajetilla la advertencia obligatoria para indicar que el producto era para exportación. La infracción era necesaria para sostener el argumento de que el producto era falsificado y evitar las multas y los impuestos debidos.

₹ Los detalles del proceso de exportación a Paraguay y Uruguay y la comparación con los consumos anuales y las exportaciones de esos países está explicado en Iglesias (2005). ${ }^{11}$ Los cigarrillos no eran reexportados legalmente desde Paraguay, que a su vez importaba de otros orígenes. Se creaba así una oferta líquida de cigarrillos en Paraguay, que en 1998 llegó a más de 35 mil millones de unidades.

$\S$ El impuesto, que es de 150\%, está todavía vigente y se extiende para todos los países de América Latina y el Caribe. 
estimularon la instalación de fábricas en Paraguay, en la segunda mitad de los noventa. Adicionalmente, esas fábricas contaron con incentivos fiscales y con la perspectiva de una carga tributaria doméstica menor. A partir de 1999, la exportación brasileña no alimentó más el mercado ilegal, pasando la producción paraguaya a hacerlo, con algunas falsificaciones al comienzo, para no perder mercados ilegales ya conquistados por el cigarrillo brasileño, pero también con marcas propias (con nombres semejantes y paquetes parecidos).*

Ramos, en la síntesis de los estudios nacionales del mercado ilegal en el Mercosur, estimó que una producción anual paraguaya de aproximadamente 70 mil millones de cigarrillos se comerció de manera ilícita en 2007. ${ }^{12}$ Esa oferta equivale aproximadamente a $10 \%$ del comercio ilegal mundial de cigarrillos y una gran parte de ella se dirige a Brasil. No existen estimaciones precisas de la cantidad de cigarrillos paraguayos que entra en el país, pero el número más citado es de 1000 millones de cajetillas o 20 mil millones de unidades. En 2009, el precio promedio al consumidor del cigarrillo paraguayo era de aproximadamente 80 centavos de dólar por cajetilla, $50 \%$ inferior al precio del cigarrillo brasileño más vendido. Considerando esos precios y dichos volúmenes puede estimarse un comercio valuado en 800 millones de dólares por año, con un alto margen de intermediación, ${ }^{\ddagger}$ que puede permitir compensar los costos de transporte, los pagos necesarios para evitar ser incautados en los pasos fronterizos y en las rutas de distribución y, finalmente, obtener una rentabilidad neta considerable para las organizaciones criminales. $\$$

\footnotetext{
* Las razones de una fábrica paraguaya para evitar la falsificación son muy simples. En primer lugar, el consumidor de menores ingresos es menos apegado a marcas y su decisión es fuertemente determinada por el precio. En segundo lugar, el servicio de inteligencia de las grandes compañías multinacionales es absolutamente especializado y muy eficiente. Falsificar productos de BAT y Phillips Morris implica un riesgo mayor de ser descubierto y perseguido.

* Se supone que el precio de puerta de fábrica en Paraguay es de aproximadamente 20 centavos de dólar, ya con los impuestos domésticos paraguayos. Si esto es así, el margen de intermediación es de 600 millones de dólares por año.

$\S$ En este tema, las definiciones de cantidad, precio y márgenes de rentabilidad son hipótesis, resultado de aproximaciones parciales y conversaciones con especialistas del sector público y privado. El tema todavía más difícil de precisar es la exacta participación de las firmas productoras paraguayas en este comercio. El argumento utilizado por ellas, como en el pasado lo fue por las grandes empresas brasileñas, es que realizan una operación legal de venta con el distribuidor, que pagan la baja carga tributaria paraguaya, y que el destino final no es su responsabilidad.
}

Dilemas de política tributaria: caída de la presión tributaria, disminución de la recaudación y continuación del mercado ilegal

La existencia de un mercado ilegal con cigarrillos más baratos fue el argumento utilizado por las dos grandes empresas para presionar, al final de los años noventa, por una carga impositiva menor. El objetivo de la estrategia iniciada en 1992, de aumento del precio real y exportación a Paraguay, quedó claro: reducir la carga impositiva sobre el cigarrillo. Después de eliminar las exportaciones a Paraguay en diciembre de 1998, las autoridades brasileñas cambiaron las características del Impuesto sobre Productos Industriales (IPI) aplicado a los cigarrillos, pasando de una alícuota ad valorem única de $42.5 \%$ sobre el precio al consumidor a alícuotas fijas para cinco categorías de cigarrillos, definidas a los efectos impositivos. ${ }^{*}$ El cambio resultó en una reducción del peso del IPI en el precio final al consumidor y en la caída nominal y real de la recaudación del impuesto sobre el cigarrillo.

La figura 3 presenta el IPI efectivamente recaudado por cajetilla como proporción del precio final del cigarri1lo. Antes de 1998, la proporción era alta, pero inferior a $42.5 \%$, porque el IPI pagado por los insumos podía deducirse y porque el precio promedio del Instituto Brasileño de Geografía y Estadística (IBGE) utilizado en este trabajo no coincide exactamente con el precio promedio resultante de la recaudación impositiva. ${ }^{\ddagger}$ Después de 1999, la caída de la proporción hasta aproximadamente $20 \%$ del precio promedio refleja la política de aumento de las alícuotas fijas por debajo de la inflación, en un contexto de inflación significativa y de incrementos de los precios de los cigarrillos. También refleja cambios en el patrón de consumo de la población, en dirección a cigarrillos que tributan con alícuotas proporcionalmente menores. De esta manera, en el contexto de un programa avanzado de control del tabaquismo, el consumidor brasileño pasó, paradójicamente, de pagar más de $35 \%$ de IPI en el periodo 1994-1998, a pagar aproximadamente 20\% de impuesto selectivo en el periodo 2003-2007.

Pero no sólo fue paradójico que se disminuyera la carga tributaria de los cigarrillos en el contexto de avances en el control del tabaquismo, también lo fue que se

\footnotetext{
* Iglesias y colaboradores ${ }^{9}$ explican detalladamente las características del cambio en el impuesto.

₹ El precio promedio que reporta el IBGE es más alto en este caso, porque no captura bien el precio de los cigarrillos más baratos que muchas veces se venden en pequeñas ciudades.
} 


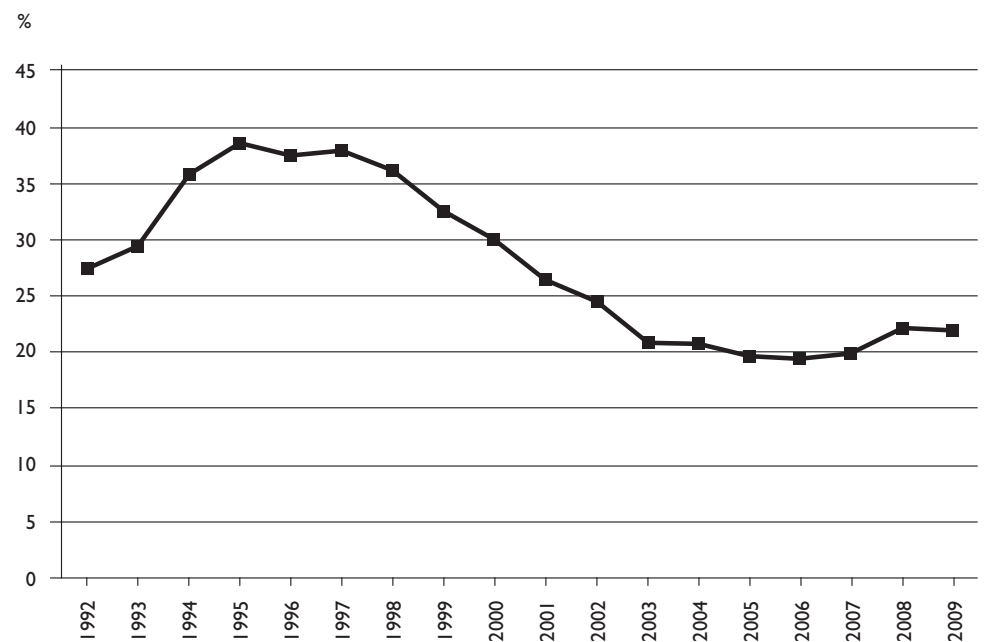

Fuentes: IPI recaudado por cajetilla = IPI recaudado total/número de cajetillas vendidas en el mercado doméstico - RFB. Precio promedio del cigarrillo - IBGE

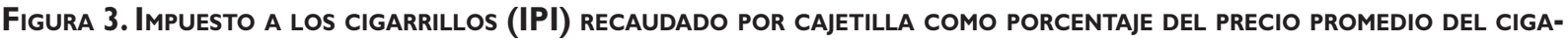
RRILLO EN BRASIL, 1992-2009

hiciera en el marco del aumento de la recaudación de impuestos federales, como el observado entre 1993 y 2008. La figura 4 presenta la evolución de la recaudación total de impuestos nacionales como porcentaje del PIB y de la recaudación de IPI sobre cigarrillos como porcentaje del PIB. En contrapartida a los retrocesos en la política tributaria aplicable a los cigarrillos, hubo avances en la administración tributaria. Las autoridades aumentaron los controles sobre la producción de cigarrillos con el objetivo de mejorar la recaudación del impuesto y evitar los mecanismos de evasión y elusión. Algunos de esos avances iniciales fueron: un nuevo tipo de declaración de impuestos, la obligación de colocar estampillas en las cajetillas, y mayores controles de los inventarios de insumos y de la cantidad producida en las fábricas.* Desde 2002 hasta 2007, las autoridades buscaron aprobar la legislación sobre un moderno sistema de control y seguimiento de la producción de cigarrillos, que fue finalmente implementado en 2008-2009.

La disminución de la carga impositiva de los cigarrillos implementada con el objetivo de reducir los atractivos del mercado ilegal resultó, como no podía ser de otra manera, en la reducción del precio real y el incremento parcial del consumo por adulto (figura 1), pero fue ineficaz para reducir significativamente la proporción de ese mercado en el consumo total. Adicionalmente,

* Iglesias y colaboradores presentan los cambios en la administración tributaria. $^{9}$ la mecánica legal utilizada para hacer el cambio en el impuesto en 1999 suscitó disputas judiciales, iniciadas por productores pequeños y de marcas baratas, lo que dificultó la administración tributaria y el aumento del impuesto en los años recientes. *

La reducción de la proporción efectiva del IPI en el precio final tenía como objetivo moderar los aumentos de precios y permitir, al mismo tiempo, mejorar los márgenes de lucro de las empresas. ${ }^{\ddagger}$ Con menores precios reales se pretendía disminuir el comercio ilegal. Sin embargo, después de 1999 y hasta 2007, la reducción significativa del precio real a través de la manipulación del impuesto no tuvo efectos significativos en el tamaño del mercado ilegal.

A partir de 2007, las autoridades parecen haber percibido que la estrategia de 1999 estaba agotada. Esto es así, pues después de haber reducido la carga de impuestos, inducido la disminución del precio real, y aumentado los controles sobre la producción doméstica,

\footnotetext{
* Las empresas menores alegaban que el cambio en la forma del impuesto se debía haber hecho con un instrumento jurídico superior. Entraron en la justicia contra el impuesto, porque además argumentaban que la alícuota fija aumentaba la carga tributaria para los cigarrillos más baratos. Estas controversias deben haber pesado en las decisiones de demorar los ajustes y hacerlos por debajo de la inflación.

* El cambio del impuesto en 1999 y la falta de ajustes de la alícuota fija, facilitó a las empresas la recomposición de sus márgenes de lucro a través del aumento moderado de los precios, tarea que siempre resulta difícil con el impuesto ad valorem.
} 
el mercado ilegal no se había alterado notoriamente hasta aquel año. La estrategia de combate debe ser otra, pues la oferta ilegal en la presente década no es sensible a ninguna de esas medidas, ya que se trata de producción ilegal proveniente del otro lado de la frontera, que no paga ningún tipo de impuestos o paga la baja carga tributaria paraguaya, no tiene ningún control sobre las cantidades producidas y tributadas lo que facilita la evasión, y opera con costos de producción antes de impuestos sensiblemente menores que los brasileños.

Algunas medidas implementadas a raíz del cambio en la percepción de las autoridades tributarias brasileñas merecen ser destacadas: aumento del IPI como proporción del precio final en 2008 y 2009 (figura 3), aumento de las alícuotas fijas del IPI por arriba de la inflación en 2009, y aumento de otras contribuciones que gravan los cigarrillos. ${ }^{*}$ Actualmente, la incidencia conjunta de los impuestos indirectos a los cigarrillos es de alrededor de $60 \%$ del precio al consumidor. Estas medidas reflejan la conciencia de que la política de precio real bajo llegó muy lejos sin lograr muchos resultados concretos, pero también obedecen a otras razones como la caída de la recaudación obtenida de los cigarrillos y la necesidad de compensar desgravaciones de otros impuestos hechas durante la crisis de 2008. Finalmente, las autoridades parecen haber entendido que las organizaciones que trabajan en la ilegalidad no pueden ser combatidas con

* En mayo de 2009 las autoridades aumentaron el porcentaje de dos contribuciones que gravan los cigarrillos de $6.45 \%$ a casi $11 \%$ del precio al consumidor. menores impuestos, pero sí con medidas específicas que corrijan las fallas que permiten ese comercio ilegal.

\section{El caso mexicano}

\section{Evolución del consumo por adultos (1981-2009)}

En las últimas tres décadas, el consumo legal de cigarrillos presenta una tendencia claramente decreciente (figura 5). A principios de los ochenta, el consumo promedio era de 1428 cigarrillos por adulto, 10 años después había disminuido a 1016 cigarrillos, y 20 años después era de sólo la mitad de la cifra inicial, 761 cigarrillos por adulto en promedio. En 2008, el consumo fue de 529 cigarrillos por adulto.

Las estimaciones sobre consumo ilegal de cigarrillos difieren, pero aun considerando esas cifras, se observa una reducción en el consumo total en años recientes. Una fuente señala que la suma del consumo legal e ilegal se redujo de 564 cigarrillos per cápita en 1994 a 484 cigarrillos en $2006 .{ }^{13}$ Otra fuente indica que esta suma se redujo de 720 cigarrillos por adulto en 2003 a 499 cigarrillos en $2008 .{ }^{14}$

La principal variable vinculada con la reducción del consumo de cigarrillos son los precios de estos productos (figura 5). Se estima que por cada aumento del precio real de los cigarrillos de $10 \%$, el consumo se reduce $5.2 \%$ en promedio, en México. ${ }^{15}$ Esta reducción se debe en parte a reducciones en la cantidad que consumen los fumadores, y en parte a reducciones en la prevalencia (o incrementos en la cesación).

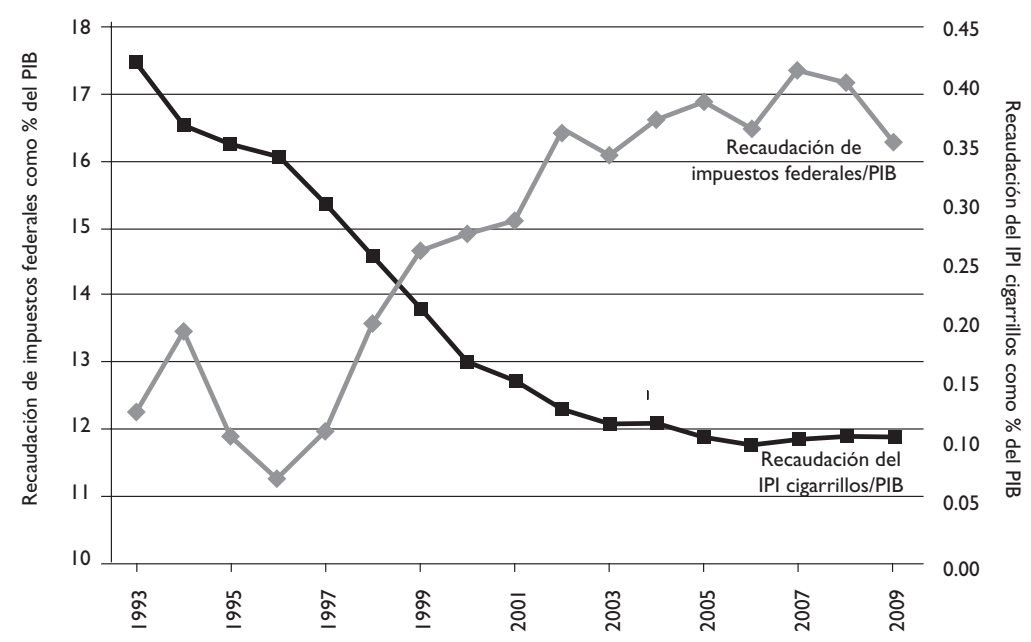

Fuente: RFB

Figura 4. Recaudación de impuestos federales y del impuesto a los cigarRillos (IPI) en Relación Con el PIB en BRASIL, 1993-2009 


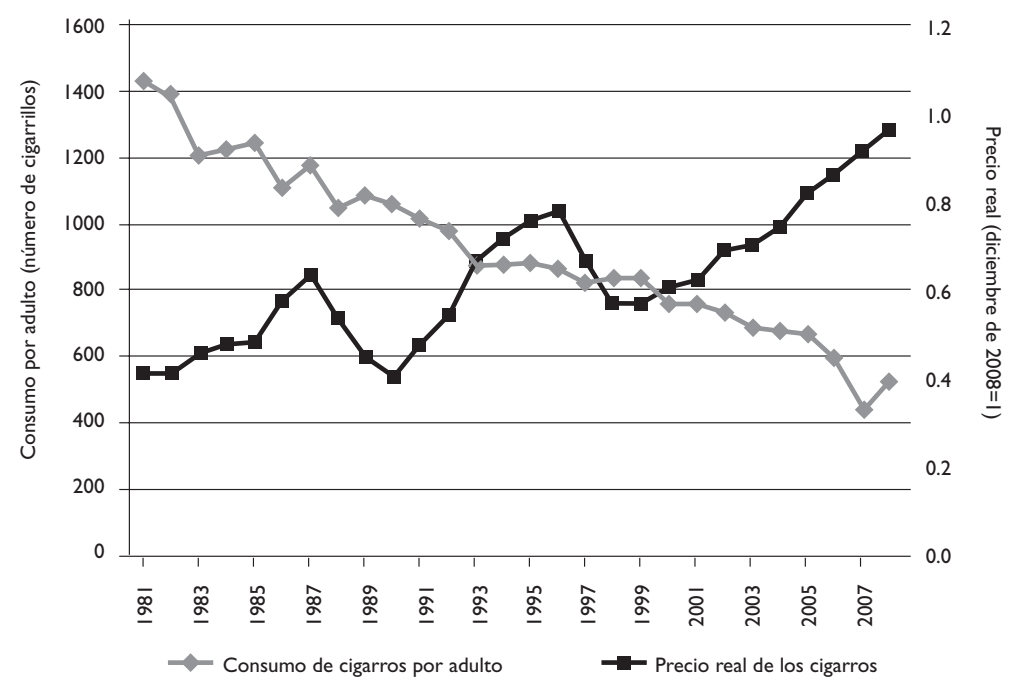

Fuentes: Precio real = índice de precios de los cigarrillos /Índice Nacional de Precios al Consumidor (INPC) - Banco de México. Consumo por adulto = (producción + importaciones - exportaciones)/ población de 15 años y más - Instituto Nacional de Estadística y Geografía (INEGI), Secretaría de Economía

Figura 5. Consumo legal por adulto y precio Real del cigarrillo en México, | 98 |-2008

Es probable que otras medidas para el control del tabaquismo también hayan contribuido a la reducción del consumo de cigarrillos, en especial en la última década, en la cual se han tenido mayores avances. En 2004, por ejemplo, año en el que México ratificó el CMCT, se prohibió la publicidad en radio y televisión.*

Impuestos, precio real y recaudación en las últimas décadas

La evolución de los precios de los cigarrillos se relaciona estrechamente con la de los impuestos (figura 6). ${ }^{\ddagger} \mathrm{A}$ mediados de los años noventa, por ejemplo, los precios reales se redujeron después de implementada una reducción de los impuestos, mientras que en la última década,

* Actualmente, la legislación relacionada con el control del tabaquismo a nivel federal, con excepción de la concerniente a los impuestos, se concentra en la Ley General para el Control del Tabaco (LGCT) vigente desde agosto de 2008.

₹ La medida en la que los productores transfieren los impuestos a los consumidores depende de la estructura del mercado. En un país como México, en el que dos industrias controlan más del $90 \%$ de la producción de cigarrillos y existe cierto nivel de colusión entre ellas, ${ }^{16}$ es de esperarse que los impuestos se trasladen al menos en parte al consumidor. ${ }^{6}$ En 2007, por ejemplo, parece que los productores trasladaron casi todo el incremento del impuesto a los consumidores, aunque en menor medida para el caso de las marcas nacionales (tradicionalmente más baratas). Ver: Saenz de Miera B, Thrasher JF, Chaloupka FJ, Waters H, Hernandez-Avila M, Fong GT. Self-reported price, consumption and brand switching of cigarettes in a cohort of Mexican smokers before and after a cigarette tax increase. En prensa. tanto los precios como los impuestos a los cigarrillos se han incrementado paulatinamente.

El Impuesto Especial sobre Producción y Servicios (IEPS), que grava a los productos de tabaco, se incrementó de $100 \%$ sobre el precio de venta al detallista en 2001 a 105 , 107 y 110\% en 2002, 2003 y 2004, respectivamente, para el caso de los cigarrillos con filtro.* Al mismo tiempo, la tasa vigente para los cigarrillos sin filtro se incrementó de $20.9 \%$ en 2001 a 60, 80, 100 y $110 \%$ en 2002, 2003, 2004 y 2005, respectivamente. De esta manera, desde 2005, todos los cigarrillos enfrentan la misma tasa impositiva. A finales de 2006, se aprobaron nuevos incrementos que llevaron el IEPS aplicable a los cigarrillos a 140\% en 2006, $150 \%$ en 2007 y $160 \%$ en $2009 .^{\ddagger}$

* El precio de venta al detallista, la base gravable del IEPS, es el precio que paga el detallista al fabricante, importador o mayorista. En enero de cada año, los fabricantes e importadores deben registrar ante la autoridad fiscal una lista de precios para todos sus productos, clasificados según marca y presentación. La lista debe indicar el precio de fábrica o precio al mayorista, el precio al detallista y el precio sugerido de venta al público. Si estos precios se modifican en el transcurso del año, el fabricante o importador debe presentar una nueva lista de precios en el transcurso de los cinco días siguientes a la implementación de los cambios.

₹ La tasa impositiva que enfrentan los productos de tabaco hechos enteramente a mano se ha mantenido menor a la del resto de los productos de tabaco (en 30.4\%) con la finalidad de proteger el empleo que genera su manufactura. Sin embargo, la mayoría de los productos hechos a mano son puros o puritos que se consumen muy poco en México, al menos en parte, porque son más caros que los cigarrillos (aún en sus presentaciones más baratas). ${ }^{14}$ 
La tasa máxima que ha alcanzado el IEPS para los cigarrillos se implementó en la segunda mitad de los ochenta. En 1986, el IEPS aumentó de 139 a 180\%. En esos años, el país atravesaba una crisis económica severa por lo que la motivación principal de ese incremento fue la necesidad de aumentar la recaudación fiscal. En la figura 7 se puede ver que la recaudación fiscal efectivamente aumentó después de ese cambio en el impuesto. En general, se observa una relación positiva entre la evolución de los impuestos y la recaudación fiscal. En el periodo de 2006 a 2008, en el que los impuestos aumentaron como se comenta arriba, la recaudación por concepto del IEPS a los tabacos labrados se incrementó 38\% en términos reales, al pasar de 18511 millones de pesos (o 1381 millones de dólares) a 25542 (o 1906 millones de dólares).*

Los productos de tabaco también pagan el Impuesto al Valor Agregado (IVA). En las últimas décadas, la tasa vigente de IVA había sido de $15 \%$ sobre el precio de venta al consumidor, pero en 2010 se incrementó a 16\%, excepto en las ciudades de la frontera norte en donde pasó de 10 a $11 \%$.

La última reforma a la Ley del IEPS se aprobó a finales de 2009. Con esta reforma se logró implementar una alícuota fija, adicional a la tasa ad valorem vigente

* Todas las cifras en términos reales de este apartado están expresadas en precios de diciembre de 2008. Por lo tanto, el tipo de cambio que se utilizó para convertir las unidades a dólares es el vigente en ese mes según información del Banco de México, 13.4 pesos por dólar. de $160 \%$. En el año en curso, la alícuota fija vigente es de 0.04 pesos por cigarrillo, es decir, 0.8 pesos (o alrededor de 0.06 dólares) por cajetilla de 20 cigarrillos. Para los siguientes tres años se estipula que esta alícuota aumentará a $0.06,0.08$ y 0.1 pesos por cigarrillo.

Como resultado del incremento de los precios en la última década, los cigarrillos se han vuelto menos asequibles, aunque no menos que en 1995. Esto quiere decir que el ingreso promedio por persona en 2008 permite comprar prácticamente la misma cantidad de cigarrillos que en 1995. La figura 8 muestra la relación entre el precio de los cigarrillos y el ingreso de la población, medido este último por el producto interno bruto per cápita.* Los puntos menores a 1 indican los años en los que los cigarrillos se han vuelto menos asequibles que en el año de referencia, 1993. Éste es el caso de los últimos cuatro años, en los que los incrementos de los precios han sido mayores al crecimiento del ingreso. Lo mismo se observa entre 1994 y 1996, aunque en dicho periodo la caída de los ingresos por la crisis económica del momento también contribuyó a la reducción de la capacidad de compra de cigarrillos. Se ha estimado que un aumento (reducción) de los ingresos de 10\% incrementa (reduce) el consumo en $4.9 \%$ en promedio, en México. ${ }^{15}$

* Blecher y Van Walbeck han usado esta relación entre precio y PIB per cápita para analizar qué tan asequibles son los cigarrillos en varios países a través del tiempo, aunque el indicador que utilizan es distinto al que se presenta en la figura 8 , ya que ellos se refieren al PIB per cápita necesario para adquirir 100 cajetillas. ${ }^{17,18,19}$

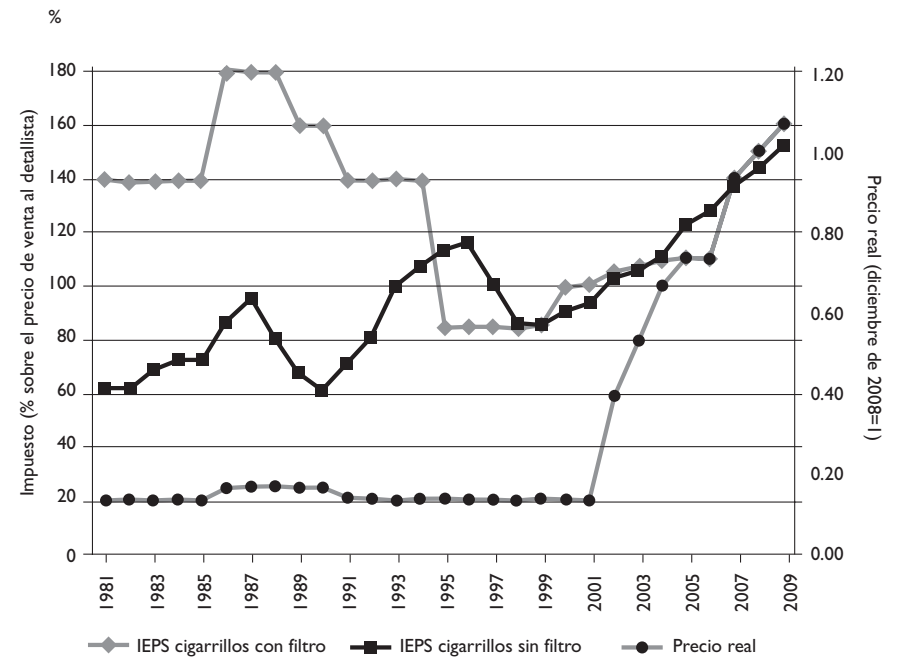

Fuentes: Impusto a los cigarrillos (IEPS) - Ley del IEPS, Diario Oficial de la Federación. Precio real - Banco de México

Figura 6. Impuesto a los cigarrillos (IEPS) y precio real de los CigarRiLlos en México, I 98 I-2009 


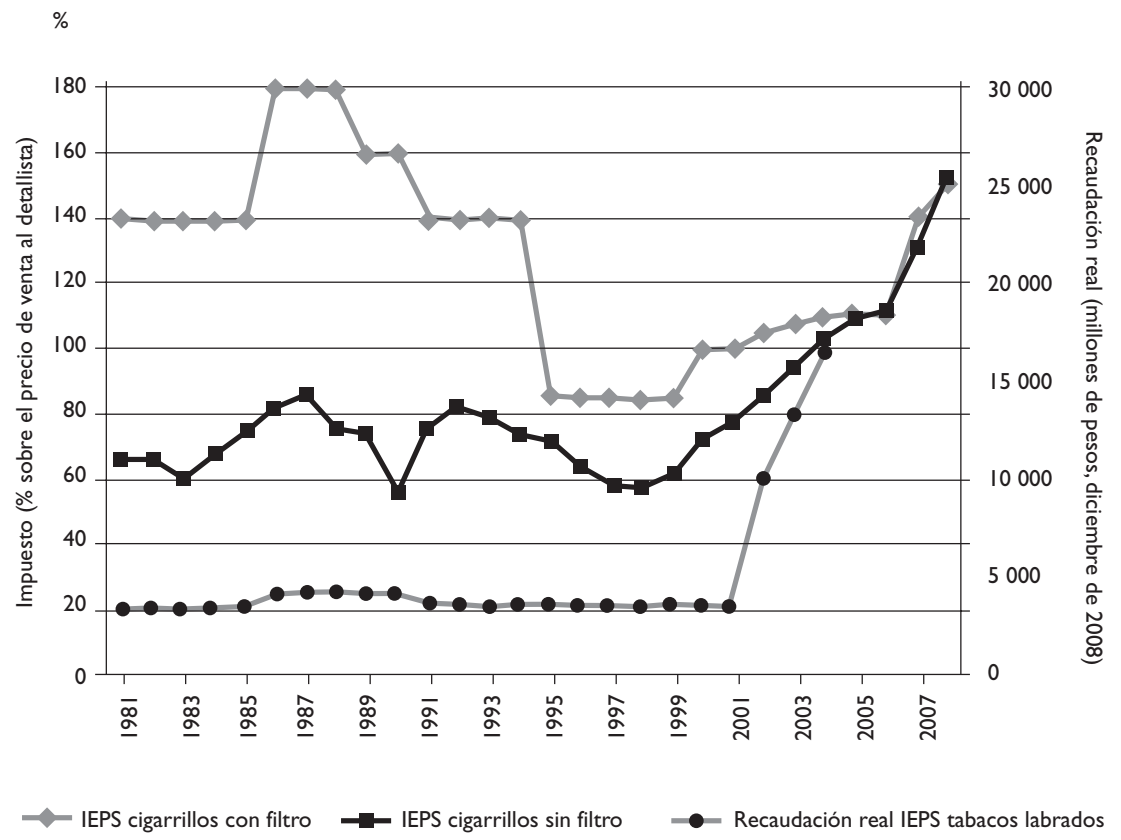

Fuentes: Impuesto a los cigarrillos (IEPS) - Ley del IEPS, Diario Oficial de la Federación. Recaudación fiscal por concepto de IEPS a los tabacos labrados - Secretaría de Hacienda y Crédito Público

Figura 7. Impuesto a los cigarrillos (IEPS) y recaudación fiscal en México, 198 I-2008

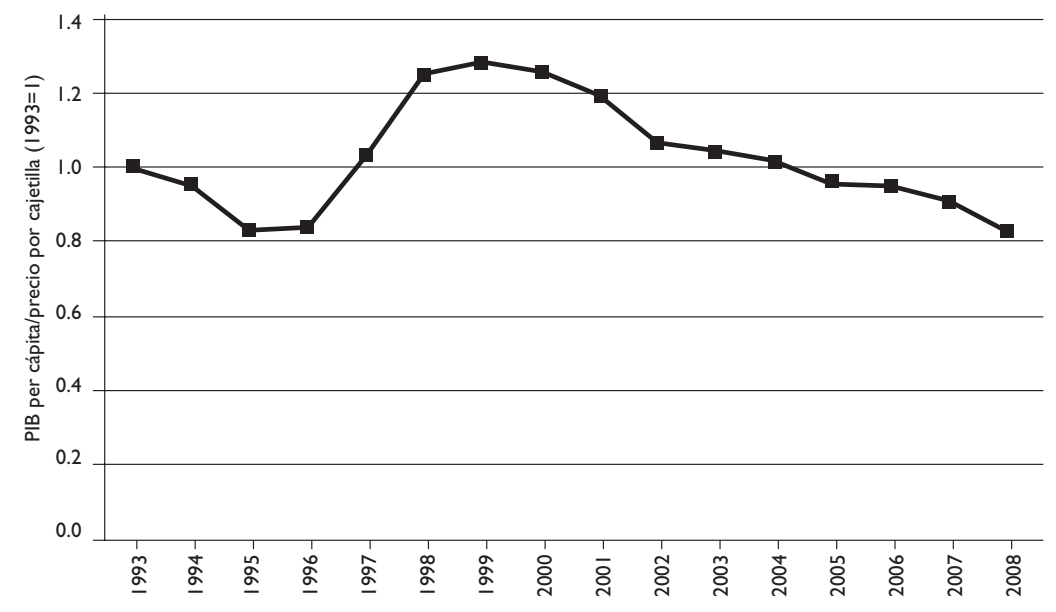

Fuentes: Precio - Banco de México. Ingreso promedio de la población = Producto Interno Bruto (PIB) per cápita - INEGI

Figura 8. Relación entre el precio de los cigarrillos y el ingreso promedio de la población en México, 19932008 
Retos de política tributaria actual: incremento

de la incidencia impositiva y control del comercio ilícito

De acuerdo con la OMS, los esquemas impositivos que comprenden alícuotas fijas se consideran los más apropiados para proteger la salud pública pues resultan en incrementos de precios más altos y permiten reducir las diferencias de precios entre marcas. ${ }^{3}$ En este sentido, la reforma recientemente implementada en México constituye un avance. Sin embargo, las características de la misma plantean retos importantes para los próximos años.

En primer lugar, las alícuotas fijas deben ser ajustadas por la inflación para evitar que pierdan su valor y que los cigarrillos se vuelvan relativamente más baratos. En la ley vigente no se consideran este tipo de ajustes, a pesar de que son comunes para otro tipo de pagos tributarios fijos. Por lo tanto, con una inflación anual esperada de $4.3 \%$, el valor real de la alícuota de 2 pesos por cajetilla planteada para 2013 será en realidad de sólo 1.7 pesos.

Más importante aún es que a pesar del último incremento la incidencia de los impuestos a los cigarrillos en México es todavía relativamente baja. En 2010, la incidencia del IEPS fijo es de $2.8 \%$ del precio final real aproximadamente, la del IEPS ad valorem es de $46.2 \%$, y la del IVA es de $13.8 \%{ }^{*}{ }^{* 20}$ En consecuencia, la incidencia conjunta del IEPS y el IVA es de $62.8 \%$ del precio final de los cigarrillos, es decir, menor a la de otros países de la región, como Argentina, Chile y Uruguay, y menor a la de algunos países desarrollados en los que alcanza cerca del $75 \% .3,21,22,23$ Con la legislación vigente se estima que el precio real de venta al público será apenas 9\% más alto en 2013 que en 2009, y el consumo sólo 4\% más bajo, por lo que el efecto en términos de salud pública será lento y limitado. Para alcanzar una incidencia de 75\% del precio final, la alícuota fija tendría que ser de 17 pesos por cajetilla (o 1.3 dólares), con lo que se tendría un precio de venta al público promedio de 48.6 pesos aproximadamente (o 3.6 dólares), ${ }^{20}$ esto es, aún menor al de las marcas más vendidas en Canadá y Estados Unidos (6.48 y 4.58 dólares a precios de 2008, respectivamente), pero similar al de la marca más vendida en Belice (3.5 dólares). Como hasta ahora, ese precio sería mayor al de

\footnotetext{
* Se estima que el precio real de venta al público por cajetilla es de 27.73 pesos (o 2.07 dólares) en promedio, en 2010. Este precio está compuesto por: 1) el precio de venta al detallista promedio de 8.01 pesos (el cual constituye la base gravable del IEPS e incluye los costos y ganancias del productor y del mayorista), 2) el IEPS ad valorem de $160 \%$ equivalente a 12.82 pesos, 3 ) la alícuota fija de IEPS de 0.77 pesos (es decir, el monto vigente de 0.8 descontado por la inflación anual esperada de $4.3 \%$ ), 4) el margen del detallista de $10.72 \%$ equivalente a 2.31 pesos, y 5) el IVA de $16 \%$ equivalente a 3.83 pesos. $^{20}$
}

las marcas más vendidas en Guatemala (1.29 dólares) y el resto de los países de Centroamérica. ${ }^{3}$ La reducción esperada en el consumo con una alícuota fija de 17 pesos es de $42 \%$. ${ }^{*}, 20$

Cuando se discutió la última modificación del impuesto a los productos de tabaco en el Congreso, se presentaron propuestas que planteaban incrementos mayores a los efectivamente aprobados. Una de esas propuestas (por parte del Senado) consistía en implementar en el año en curso el incremento que en la legislación actual tendrá vigencia hasta 2013. De esta manera, aunque el efecto sobre el consumo sería igualmente limitado, al menos habría sido más inmediato. La posible caída del empleo que generan la manufactura de cigarrillos y el cultivo de hoja de tabaco, así como el incremento del comercio ilegal, fueron los principales argumentos que se utilizaron para frenar aumentos mayores en los impuestos.

No obstante, el empleo en el sector agrícola y manufacturero que genera el tabaco en México es muy reducido. La producción de hoja de tabaco contribuye apenas con cerca del $0.1 \%$ del empleo total en el sector primario, mientras que la manufactura de cigarrillos contribuye con $0.4 \%$ del empleo total en el sector manufacturero. ${ }^{16,20}$ El caso de la producción de hoja de tabaco es particularmente interesante, ya que la superficie cosechada ha disminuido drásticamente en las últimas décadas, y por lo tanto, la producción y el empleo en el sector. Pero esta reducción parece más relacionada con la estrategia de la industria de incrementar las importaciones de hoja, que con la implementación de políticas para el control del tabaquismo, es decir, parece que la misma industria ha inducido a muchos agricultores que antes sembraban tabaco a cambiar de cultivo al dejar de comprar la hoja de tabaco nacional.

En cuanto al comercio ilegal de productos de tabaco, no se ha observado un aumento acelerado después de los últimos incrementos en los impuestos. En 2008 se estimó que el comercio ilegal de tabaco en México fue de $5.9 \%$ del consumo, ${ }^{14}$ cifra por debajo del promedio mundial $(11.6 \%) .^{24}$ De cualquier manera, es necesario considerar que futuros incrementos de los impuestos y precios posiblemente generen mayores incentivos para introducir cigarrillos ilícitos al país. Como hemos dicho, los precios de los cigarrillos (incluyendo los impuestos domésticos) en casi todos los países de Centroamérica y en varios de Sudamérica son relativamente menores que en México. ${ }^{3}$ De hecho, ya se han detectado cigarrillos

\footnotetext{
* Esta reducción en el consumo incorpora el efecto del crecimiento del ingreso, es decir, es la suma de la reducción producida por el incremento del precio y el aumento producido por el crecimiento del ingreso.
} 
ilícitos provenientes de Paraguay e incluso de China en el país. ${ }^{14}$ Sin embargo, el control y la reducción del comercio ilegal puede lograrse a través de medidas diseñadas específicamente para ello. En la última reforma a la Ley del IEPS se dio un paso en este sentido, al añadir la obligación de que los productores e importadores de cigarrillos y otros tabacos labrados (excepto los hechos enteramente a mano) impriman un código de seguridad en las cajetillas para la venta en México. En principio esta disposición entraría en vigor el 1 de julio de 2010, aunque recientemente se ha permitido no imprimir el código de seguridad durante el ejercicio fiscal de 2010. En los próximos años se tendrá que trabajar, entre otras cosas, en la conformación de un sistema de seguimiento y localización de la producción nacional y en el fortalecimiento de los controles en las fronteras.

\section{Recomendaciones}

El artículo 6 del CMCT establece una orientación general para las políticas tributarias nacionales: contribuir a los objetivos de salud reduciendo el consumo de tabaco, aunque no da ninguna orientación específica sobre las características que debe tener la política tributaria para contribuir con esos objetivos. ${ }^{2}$ Esto quiere decir que desde el punto de vista de la salud no existe hoy una fórmula con parámetros definidos para establecer el nivel y la estructura óptima del impuesto selectivo (como el IPI o el IEPS) y de otros impuestos indirectos (como el impuesto al valor agregado) que pagan los cigarrillos.

Sin embargo, las autoridades cuentan con modelos nacionales de demanda de cigarrillos legales, los cuales han mostrado que el consumo por adulto de cigarrillos depende negativamente de su precio real y positivamente del ingreso disponible real per cápita. ${ }^{9,15}$ De esta manera, un primer criterio que deben considerar es que los impuestos a los cigarrillos tienen que aumentar de forma tal que el incremento del precio real resultante permita compensar, o más que compensar, el crecimiento del ingreso disponible per cápita. Sólo así el consumo se mantendría constante o caería.

También debe considerarse la recomendación del reporte más reciente de la OMS sobre la situación de la epidemia del tabaquismo. ${ }^{4}$ A partir de las mejores prácticas observadas en el ámbito internacional, en este reporte se recomienda que la proporción del impuesto sobre el precio final del cigarrillo sea de entre dos terceras $(67 \%)$ y cuatro quintas partes $(80 \%)$. Los países aquí analizados tienen una incidencia impositiva menor: en México los impuestos a los cigarrillos representan aproximadamente $63 \%$ del precio al consumidor y en Brasil alrededor de $60 \%$.
En cuanto a la estructura impositiva, se tiene que las combinaciones posibles de base imponible y alícuota del impuesto selectivo al cigarrillo son muchas, reflejando la mayoría de las veces aspectos históricos de los países, pero también presiones de la industria para disminuir la carga efectiva sobre el precio final del cigarrillo. Con el objetivo de maximizar el efecto del aumento de los impuestos sobre los precios, la teoría y la experiencia en administración tributaria indican que el impuesto selectivo debe tener un componente fijo, porque la alícuota fija incentiva el aumento del precio para mejorar el margen de lucro por unidad de producto y reducir la carga tributaria en relación con el precio final. Con alícuotas ad valorem, especialmente sobre el precio final, los aumentos de precios no alteran el margen de lucro de la cadena. Por eso, los productores prefieren trabajar con precios bajos cuando se usan impuestos ad valorem, ampliando sus ganancias totales a través de las cantidades vendidas. ${ }^{*}$ Los dos países analizados han incorporado un componente fijo a la estructura impositiva de los cigarrillos, pero parece que no lo han aprovechado en toda su potencialidad. Brasil implementó un sistema de alícuota fija en 1999, pero dejó sin ajustar la alícuota por tres años y medio, a pesar de que el alza de precios acumulada en el periodo fue de casi $40 \%$. Por esto, no sorprende la caída del precio real del cigarrillo que acompañó a esa política impositiva. A partir de 2007, las autoridades tributarias comenzaron a ajustar la alícuota fija por la inflación acumulada, en la misma medida o en un porcentaje mayor (como en marzo de 2009), con lo que el precio real del cigarrillo aumentó sin que hubiera una alteración sustancial de la carga de impuestos indirectos en el precio final. En el caso de México, la alícuota fija se implementó a fines de 2009, pero es muy pequeña en relación con el precio promedio al consumidor vigente para tener un efecto significativo sobre éste.

Tres recomendaciones de política tributaria surgen de la experiencia reciente de los países analizados. En primer lugar, las alícuotas fijas deben establecerse como un porcentaje significativo del precio final para producir efectos sustantivos sobre el consumo. En segundo lugar, deben ajustarse regularmente de acuerdo con la inflación acumulada. En tercer lugar, deben ajustarse

\footnotetext{
* Cuando la alícuota ad valorem es sobre el precio de fábrica o el precio de venta al detallista, como en México, las empresas tienden a mantener deprimida la base gravable y a aumentar el margen de intermediación y el precio final, especialmente si participan en el proceso de distribución. Esto tiene consecuencias negativas sobre la recaudación, pero puede estimular mayores aumentos en el precio real.
} 
para compensar, o más que compensar, el crecimiento del poder adquisitivo del ingreso personal.

La implementación de la política tributaria sobre los cigarrillos también tiene otros dilemas. Un crecimiento sustancial y brusco del precio real de los cigarrillos legales o una caída muy pronunciada de la relación entre el ingreso disponible y el precio puede llevar a un aumento de la demanda de los sustitutos próximos del cigarrillo legal, como el tabaco picado o para armar y los cigarrillos de origen ilegal o sin impuestos. Para evitar esa sustitución e inducir una reducción del consumo total de productos de tabaco, los impuestos de los sustitutos legales deben ser similares a los de los cigarrillos legales. Cualquier política tributaria de cigarrillos legales que no tome en cuenta los precios o el acceso a los sustitutos próximos tendrá posibilidades limitadas de disminuir el consumo total de los productos de tabaco.

La cuestión más complicada es cómo hacer con el producto ilegal o sin impuestos. La experiencia brasileña indica que el precio del cigarrillo ilegal tiende a aumentar en una proporción semejante al del cigarrillo legal (el precio relativo tiende a mantenerse constante), pero la caída del ingreso disponible en términos de cigarrillos legales estimula la sustitución. El problema de Brasil cuando esta caída ocurre es que hay oferta disponible de cigarrillos ilegales y es de fácil acceso para la población.

Sin embargo, cuando hay oferta ilegal disponible y de fácil acceso, la política tributaria no es el instrumento adecuado para controlar y reprimir el mercado ilegal de los productos de tabaco, pues esta política actúa sobre dos variables que determinan la demanda del mercado ilegal, el precio relativo del cigarrillo legal e ilegal y el poder adquisitivo en términos de cigarrillos del ingreso disponible, pero hay otras variables que afectan el tamaño y las características del mercado de cigarrillos sin impuestos en un país. A partir de 1999, Brasil intentó bajar el precio relativo del cigarrillo, legal e ilegal, con una política de reducción de la carga tributaria efectiva y de no aumento de la alícuota fija, lo que permitió que creciera el ingreso disponible de los más pobres en términos de cigarrillos legales, pero no hubo efectos significativos sobre el mercado ilegal. Es decir, Brasil intentó actuar solamente sobre dos de las variables que afectan la demanda de cigarrillos ilegales.

Una vez instalado el mercado ilegal, la cuestión es cómo reducir los incentivos y el espacio para el aumento de la oferta ilegal y limitar la facilidad con que se realizan las transacciones. No hay que abaratar el cigarrillo legal como hizo Brasil, hay que controlar y encarecer la oferta de los sustitutos ilegales de los cigarrillos.

El artículo 15 del CMCT recomienda dos estrategias para combatir el mercado ilegal: implementar y desarro- llar legislación nacional relacionada con el tema, y, como se trata de fenómenos que normalmente involucran a más de una jurisdicción tributaria y administrativa, realizar acuerdos subregionales, regionales y globales. ${ }^{2}$

En materia de legislación nacional, el artículo 15 recomienda la colocación de una marca o indicación en la cajetilla, paquete o caja para identificar el origen del producto, y para que, de conformidad con la legislación nacional y los acuerdos bilaterales y multilaterales pertinentes, auxilie "a las Partes a determinar el punto de desviación y a vigilar, documentar y controlar el movimiento de los productos de tabaco y su situación legal". En esa misma línea de control de los desvíos ilegales de la oferta, el artículo recomienda la implantación de un sistema de seguimiento y localización (tracking and tracing regime) para monitorear la trayectoria del producto y asistir en la investigación del comercio ilícito. Adicionalmente, el artículo 15 recomienda fortalecer la legislación con sanciones y penalidades apropiadas contra el comercio ilegal, destruir de forma adecuada los productos de tabaco y equipamientos de producción incautados, el licenciamiento para controlar y regular la producción y distribución de los productos de tabaco, así como fiscalizar los flujos de comercio.

Para coordinar y organizar la cooperación en el combate al mercado ilegal, la Conferencia de las Partes del CMCT ha avanzado un escalón adicional al determinar la realización de negociaciones en torno de un protocolo internacional sobre comercio ilícito. Estas negociaciones se están llevando a cabo actualmente, con el objetivo de especificar algunas de las medidas mencionadas en la esfera de adecuación de la legislación nacional, así como el contenido de la cooperación internacional.

En México apenas se han comenzado a sentar las bases de lo que podría constituir un sistema de seguimiento de los productos de tabaco, por lo que en los próximos años se tendrá que trabajar en su fortalecimiento y políticas afines. Brasil ha logrado implementar un sistema de seguimiento y localización y avanzó en el control de la producción ilegal o sin impuestos de origen doméstica, pero gran parte de la producción ilegal proviene de un territorio vecino, con bajos impuestos y con debilidades notorias de fiscalización y control de la producción. Por el lado brasileño, existen debilidades y dificultades en el control de las fronteras, aunque se haya avanzado significativamente en los últimos años. Las medidas apropiadas en el caso brasileño no son sólo el control de la producción nacional, el país debe avanzar en la segunda estrategia: la cooperación regional para el combate al contrabando. Esto permitiría fortalecer la administración y fiscalización tributaria en Paraguay, 
controlar mejor el movimiento de productos, intercambiar informaciones con las autoridades vecinas y cooperar en las investigaciones y en los procesos penales.

\section{Declaración de conflicto de intereses}

Declaramos no tener conflicto de intereses.

\section{Referencias}

I.World Health Organization. Global Health Risks. Mortality and burden of disease attributable to selected major risks. Ginebra:WHO, 2009. 2. World Health Organization. Convenio Marco de la OMS para el Control del Tabaco. Ginebra:WHO, 2003.

3. World Health Organization.WHO report on the global tobacco epidemic, 2008:The MPOWER package. Ginebra:WHO, 2008.

4. World Health Organization. WHO report on the global tobacco epidemic, 2009: Implementing smoke-free environments. Ginebra: WHO, 2009.

5. Jha P, Chaloupka F. Curbing the epidemic. Governments and the Economics of Tobacco Control.Washington:The World Bank, 1999. 6. Chaloupka FJ, Hu T,Warner KE, Jacobs R, Yurekli A. The taxation of tobacco products. En: Jha P, Chaloupka FJ, eds. Tobacco Control in Developing Countries. Washington DC:The World Bank, 2000:237-272.

7. Instituto Brasileiro de Geografia e Estadistica (IBGE). Pesquisa de Tabagismo (PeTab). IBGE, Brasil, 2009.

8.Villalobos A, Rojas R. Consumo de tabaco en México. Resultados de las Encuestas Nacionales de Salud 2000 y 2006. Salud Publica Mex 2007;49(supl 2):SI47-SI54.

9. Iglesias R, Prabhat J, Pinto M, Da Costa e Silva VL, Godinho J.Tobacco Control in Brazil,Washington:The World Bank. Health, Nutrition and Population Working Paper, 2007.
10. Iglesias R. La evolución del mercado negro en Brasil. Framework Convention Alliance Bulletin 2008;75:2-4.

I I. Iglesias R. O Controle do Tabaco nos países do Mercosur e associados: o caso do Brasil.Washington: OPS, 2005.

12. Ramos A. Illegal trade in tobacco in the Mercosur countries. Framework Convention Alliance, 2009.

13. ERC Group.World Cigarettes I.The 2007 Survey:Americas. Suffolk: ERC Group Ltd, 2007.

14. Euromonitor International.Tobacco in Mexico. London: Euromonitor International, 2009.

15. Jimenez-Ruiz JA, Saenz de Miera B, Reynales-Shigematsu LM,Waters HR, Hernandez-Avila M. The impact of taxation on tobacco consumption in Mexico. Tob Control 2008; 17:105-I I0.

16. Saenz de Miera B, Jimenez-Ruiz JA, Reynales-Shigematsu LM.The Economics of Tobacco in Mexico. Cuernavaca: Instituto Nacional de Salud Pública, 2007.

17. Blecher EH,Van Walbeek CP.An international analysis of cigarette affordability. Tob Control 2004;13:339-346.

18. Blecher EH,Van Walbeek CP.An analysis of cigarette affordability. Paris: International Union Against Tuberculosis and Lung Disease, 2008.

19. Blecher EH,Van Walbeek CP. Cigarette affordability trends: an update and some methodological comments. Tob Control 2009; I8:167-175. 20.Waters H, Saenz de Miera B, Ross H, Reynales-Shigematsu LM. La Economía del Tabaco y los Impuestos al Tabaco en México. París: Unión Internacional contra la Tuberculosis y Enfermedades Respiratorias, 2010. 21. González-Rosada M. Economía del Control del Tabaco en los países del Mercosur y Estados Asociados. Argentina: 1996-2004, Washington: Organización Panamericana de la Salud, 2006.

22. Debrott D. Economía del Control del Tabaco en los países del Mercosur y Estados Asociados. Chile,Washington: Organización Panamericana de la Salud, 2006.

23. Ramos A. Economía del Control del Tabaco en los países del Mercosur y Estados Asociados. Uruguay, Washington: Organización Panamericana de la Salud, 2006.

24. Shafey O, Eriksen M, Ross H, Mackay J.The Tobacco Atlas. Atlanta: American Cancer Society, 2009. 